NuMI-L-524

$8 / 24 / 99$

\title{
MINOS Far-Detector Coil Design
}

\author{
J.K. Nelson ${ }^{\mathrm{a}}$, J. Kilmer ${ }^{\mathrm{b}}$ \\ ${ }^{a}$ Dept. of Physics, College of William \& Mary, Williamsburg, VA 23187, USA \\ ${ }^{\mathrm{b}}$ Fermi National Accelerator Laboratory, Batavia, IL 60510, USA
}

\section{Introduction}

This document has been prepared for the 8/99 Conceptual Design Review of the MINOS Far Detector Coils. It's main goal is to provide a set of references to previous documents and to assemble various design drawings and engineering calculations that have not been included in previous technical memos. It also provides some background material relevant for the coil implementation. Much of the text for this document is edited from the MINOS Detectors Technical Design Report [1]

\section{Overview of the Far Detector}

The MINOS far detector will be installed a half mile underground in the Soudan mine in northern Minnesota. The 5.4-kt structure is assembled from 8-m wide, 1- inch thick octagonal steel planes. The planes are made from low carbon (1006), hot rolled steel and are toroidally magnetized. The 486 steel planes are arranged as two "supermodules" of 243 planes each, separated by a 1.5-m long gap to allow space for installation of two separate magnet coils. Each plane is hung by two "ears," which are extensions of the octagonal plane structure, similar to the hanging files in a file drawer. The steel planes have a center-to-center spacing of $5.94 \mathrm{~cm}$.

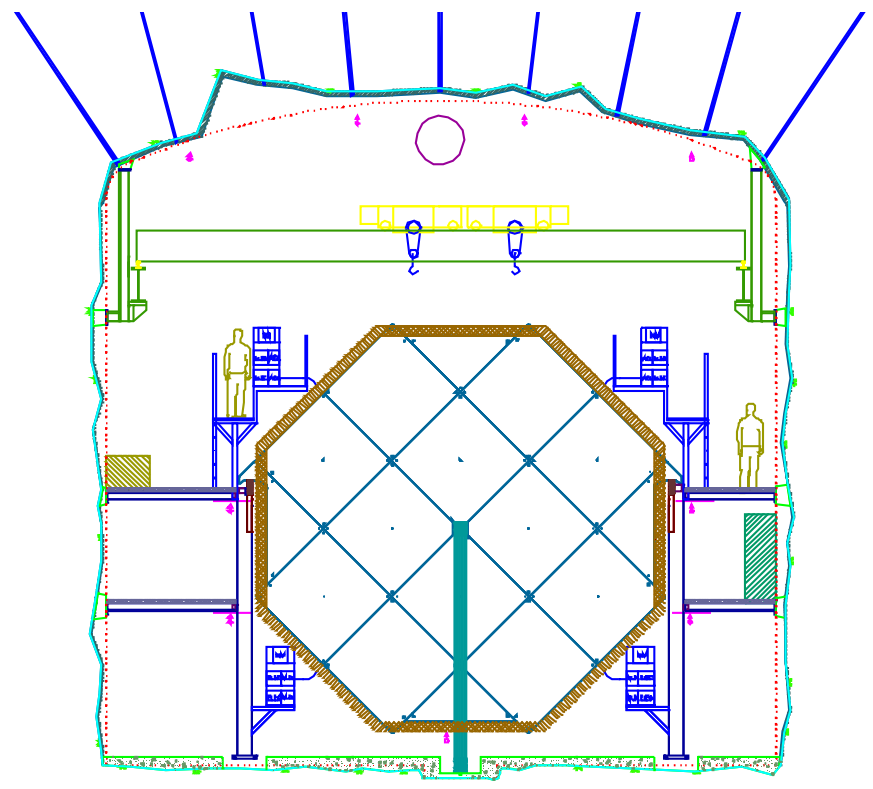


Figure 4.1: Sketch of an 8-m wide MINOS far detector steel plane hanging from the detector support rails. Also shown are the detector support structure, the side walkways, the electronics racks, and the magnet coil.

Each MINOS far detector supermodule is toroidally magnetized by a water-cooled copper-wire coil [4]. A hole at the center of each plate is provided for the coil. A coil is fabricated and installed in each supermodule after all its planes are mounted. The return leg of the coil is located in a shallow trench in the floor directly under the axis of the supermodule. This location allows access space to the bottom of the detector and minimizes interference with photodetectors and electronics.

A collar surrounds the magnet coil hole in each plane. This restraint serves two functions. It sets the spacing in the center of the planes to give the correct gap between planes, and it provides a smooth bore through which the magnet coil can be inserted. The coil hole in each steel plate is cut slightly larger than required for the collar. The collar can then be aligned laterally to take up the tolerance differences (e.g. imperfections in the plane assembly or misalignment of support rails. The ID of the collar is a $26 \mathrm{~cm}$ diameter circle.

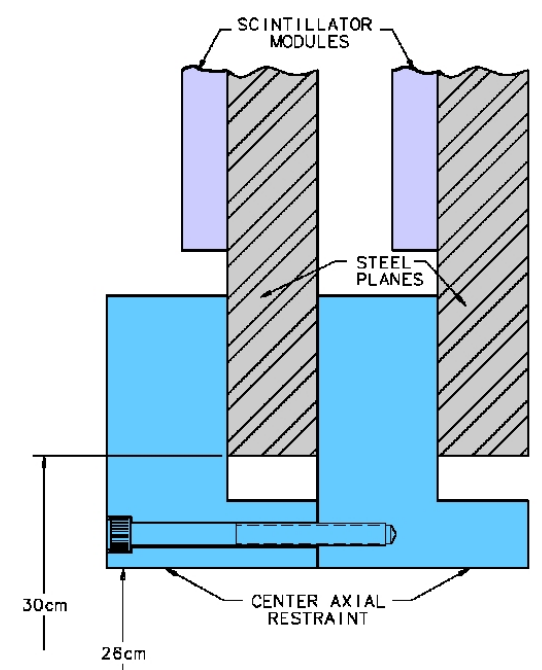

Figure 2: Section view of a steel plane coil collar showing how adjacent collars bolt together.

\section{Performance Criteria}

- Provide current of $15 \mathrm{kA}$-turns in a small package

- Minimize weight of conductor components for ease of handling

- Do not increase the temperature of the scintillation counters by more than $5^{\circ} \mathrm{C}$

- Produce a design that offers the possibility of service after coil installation

- Ensure components can be moved underground at Soudan

- Minimize the underground labor and space requirements during fabrication and installation

\section{Design and Properties of the Coils}

The magnet coils for the MINOS far detector use water-cooled copper conductor [4] to provide 15,000 A-turns for each supermodule. The far-detector coil-cooling water is provided by a local 
closed loop system; this is supplied by the MINOS Detector Outfitting Contract and is out of the scope of this review. The coil cooling system is connected to the cavern cooling system that carries the heat up the shaft to a heat exchanger on the surface, as described in MINOS Far Detector Laboratory TDR [2].

Each coil consists of a central section, in a water-cooled bore tube through the axial coil hole of a supermodule, and an air-cooled return section located in a floor trench directly beneath the central coil section. Each coil has 160 turns of $1.48 \mathrm{~cm}$ diameter (1/0 gauge) insulated, stranded copper wire which is pulled through the bore tube along with additional cooling-water tubes. Each turn includes air-cooled vertical segments that connect the bore and return sections. The turns are connected by crimping near the bottom end of one of the vertical segments. The 15,000 Aturn coil is designed to provide an average toroidal magnetic field of $1.3 \mathrm{~T}$ with minimum electrical power (and heat generation) and minimum temperature rise at the center of a supermodule (which could affect detector performance). Each coil dissipates $20 \mathrm{~kW}$ of electrical power and each coil power supply requires $29 \mathrm{~kW}$ of input power.

The far detector coils must be fabricated underground due to restrictions imposed by the existing shaft. All coil components are packaged to fit easily into the Soudan mine hoist cage (approximately $9 \mathrm{~m} \times 2 \mathrm{~m} \times 1 \mathrm{~m}$ ).

There are two identical coils for the far detector, one for each supermodule. The far detector coils provide a total of 15,000 A-turns of current in the 26-cm circular central bore of the far detector planes. These coils are designed so that their components can easily be moved down the existing hoist cage and so that they can be assembled underground without significant interruption of the assembly of subsequent detector planes.

Each far-detector coil is fabricated from 163 turns of 1/0 gauge stranded copper wire housed inside a 25-cm diameter, water-cooled, copper jacket. The outer jacket is cooled by water flowing through eight copper tubes. Seven additional tubes provide cooling near the center of the coil. A cross section of a far-detector coil is shown in Figure 3. Each of these conductors carries a current of $92 \mathrm{~A}$. In order to provide more working space under the detector, the return leg of each coil has been routed through a shallow trench in the floor of the cavern. 


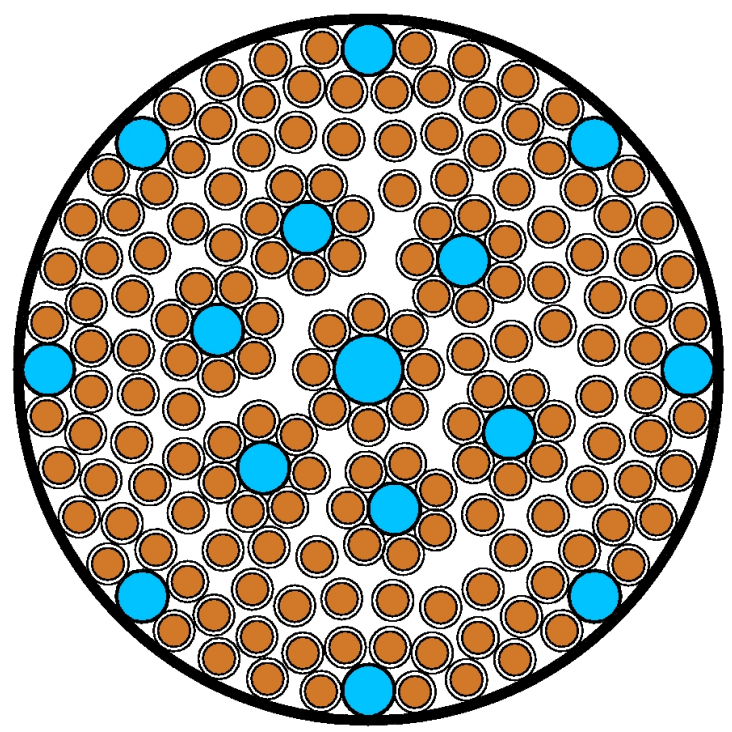

Figure 3: Sketch of a cross section of one of the far detector supermodule coils. The larger diameter circles represent the copper cooling tubes and the smaller circles are the 163 turns of $1 / 0$ gauge stranded copper wire. The outline of each of these conductors is a to-scale representation of the insulator thickness. The outer circumference is a copper jacket directly cooled by eight cooling tubes.

The thermal properties of this coil design have been evaluated using ANSYS FEA calculations [5]. The outer jacket of the coil will have a maximum increase in temperature of less than $2^{\circ} \mathrm{C}$. To gain greater confidence in these FEA calculations, they have been tested in a 1-m long by 5-cm diameter coil. The measured temperature increase in the center of the model coil, as a function of current, agreed with model calculations to better than $5 \%$.

\section{Far detector coil fabrication and installation}

The far detector coils have been designed to be assembled underground with minimal labor, fixturing and specialized tools. Coil assembly and installation will cause only a short pause (about two weeks) in the assembly of detector planes after the first supermodule is completed.

The components of the far detector coil, the conductor and cooling tubes are delivered to Soudan coiled on reels. The outer jacket is shipped in open sections small enough to fit into the hoist cage. While the last planes of a supermodule are being installed, the coil-cooling jacket is assembled. The sections are brazed together into full length half pipe, the outer cooling tubes are soldered to the inside of the outer shell, and then the halves are brazed together to form a tube.

The cooling jacket is then inserted into the completed supermodule. The flexible 1/0 gauge copper TGGT wire is single turn lengths and each conductor is labeled. The wires are then pulled through the jacket. Seven times during the winding of this coil, copper cooling tubes are unrolled and inserted into the coil. After the coil is wound electrical-distribution grade crimp connections are used to connect the separate turns of the coil. Finally, the cooling tubes are plumbed into the cavern cooling system. 
One of the advantages of this design is that after the cooling jacket has been inserted into a supermodule, the coil installation and commissioning can proceed in parallel with the assembly of the next supermodule. Another advantage is that, if repairs are needed, faulty turns can be removed and replaced.

The bore tube is constructed from sections of $25 \mathrm{~cm}$ diameter, $3.2 \mathrm{~mm}$ thick rolled copper tube with eight longitudinal chilled water tubes (2cm diameter copper refrigeration tubing) soldered to its inside surface. The bore tube is the same length as the supermodule, and provides chilled water cooling for the bore section of the coil.

The conductors are commercial grade 1/0 stranded copper building wire (TGGT) with Teflon insulation, $1.48 \mathrm{~cm}$ in diameter. The coil conductors are pulled through the bore tube in small bundles, seven of which include $2 \mathrm{~cm}$ diameter copper coolingwater tubes. The procedure disperses the cooling tubes as uniformly as possible in order to maintain a homogenous temperature profile inside the coil. The bore tube is packed tightly to maintain good heat transfer between the conductor turns and the cooling tubes. As the turns are installed in the bore tube, the remaining length is placed in fixtures provided for support of the horizontal and vertical return sections of the coil. The fixtures along the vertical legs of the coil provide space between layers of the coil for air circulation. Connections between the turns of the two coil sections and the power supply are made with a crimping tool and then insulated. The bore tube is designed to limit the temperature rise of the steel planes and scintillator near the coil hole to less than $2^{\circ} \mathrm{C}$. The maximum operating temperature in all sections of the coil is expected to be less than $50^{\circ} \mathrm{C}$, compared to the $200^{\circ} \mathrm{C}$ conductor temperature rating. The Teflon conductor insulation permits normal operation even if the return coil floor trench should be filled with water as the result of an accident. The chilled water for the cooling tubes removes heat from the bore and return sections directly to the cavern cooling system heat exchanger.

\section{Coil Cooling Systems}

The water cooling system must maintain the magnet coils at less than $5^{\circ} \mathrm{C}$ above ambient to ensure that scintillator systems are not prematurely aged. Since no current is carried in the cooling tubes in the far-detector coil, the cooling system can operate using ordinary water. This means that the cooling pipes can be directly plumbed into the cavern cooling system. This system transfers heat, via multiple pumping stations, to a surface cooling tower. The far-detector water cooling system will carry about $19 \mathrm{gpm} / \mathrm{coil}$ and is designed to carry off as much as $25 \mathrm{~kW}$ of heat per far detector supermodule.

\section{Power Systems}

The far detector has two PEI $20 \mathrm{~kW}$ trim switching power supplies, one for each supermodule coil. The input power is $480 \mathrm{~V}, 3$ phase, and the output is $92 \mathrm{~A}$ at $190 \mathrm{~V}$. Both supplies are equipped with input and output filters to reduce electronic noise pickup and have remote readout and remote control capabilities. The current will be monitored using shunts.

Both coils will be instrumented with thermal sensors, resistance bridges, and interlocks to detect possible hot spots in the event of local mechanical or electrical failure. 


\section{Coil Installation Schedule and Costs}

The coil for the first supermodule will be installed early 2002 and the coil for the second supermodule will be installed in early 2003. It takes approximately two weeks to install each magnet coil.

A cost estimate for the coil materials is summarized in Reference [4]. The cost is dominated by the purchase of the conductor.

\section{Coil Prototyping Plans}

In FY98 a small diameter test coil was produced to validate the thermal model described in Reference [5]. A full-current non-cooled prototype coil was produced for the MINOS Far detector one-plane prototype (1PP) in FY99. This device was used for magnetics studies in the 1PP.

In FY00 two additional prototypes will be constructed. The conductor for the first far-detector coil will be purchased and delivered to Fermilab for use in these prototyping efforts. The first prototype will be a pulling prototype to understand the issues associated with getting the conductors and cooling tubes down the bore of the detector. The prototype will be the length of the actual coils but will have a smaller diameter than the final coils. The second prototype will be a full-diameter prototype to understand the issues associated with fabricating the cooling jacket and installation of the conductor. This prototype will use the final jacket design but will be shorter then the final coils. After completion of the prototypes the conductor will be spooled and shipped to Soudan for installation in the first supermodule.

\section{References}

[1] The MINOS Collaboration, "The MINOS Detectors Technical Design Report," October 1998, Fermilab report NuMI-L-263.

[2] The University of Minnesota, CNA Consulting Engineers, Ericksen-Ellison Associates, Inc., and Miller-Dunwiddie, Inc., 'MINOS Far Detector Laboratory Technical Design Report (Including Basis of Estimate \& WBS) for Cavern Construction, Cavern Outfitting \& Detector Outfitting," September 1998, Fermilab report NuMI-L-263.

[3] The Fermilab NuMI Group, "MINOS Cost and Schedule Plan," September 1998, Fermilab report NuMI-L-342.

[4] J. Kilmer et al., “Basis of Estimate for the MINOS Far Detector Magnet Coils," September 1998, Fermilab report NuMI-L-404.

[5] Z. Tang, "'Thermal analysis of the MINOS far detector magnet coil," September 1998, Fermilab report NuMI-L-413. 